\title{
Aplicación del método del caso: Una integración entre la teoría y la práctica en cirugía
}

\section{Application of the Method Case: Integration Between the Theory and the Surgical Practice}

\author{
Jorge Oviedo Quirós DDS, OMFS, MScํㅜ Patricia Hernández Rivera DDS, MAE² \\ 1. Máster en Ciencias Odontológicas, Especialista en Cirugía Oral y Maxilofacial. Hospital Nacional de Niños Dr. \\ Carlos Sáenz Herrera, Departamento de Ciencias Diagnósticas y Quirúrgicas, Facultad de Odontología, \\ Universidad de Costa Rica, Costa Rica \\ 2. Máster Administración de Instituciones Educativas, Departamento de Ciencias Diagnósticas y Quirúrgicas, \\ Facultad de Odontología, Universidad de Costa Rica, Costa Rica \\ Autor para correspondencia: Dr. Jorge Oviedo Quirós - oviedo_05@yahoo.com \\ Recibido: 13-XI-2014 \\ Aceptado: 14-IX-2015 \\ Publicado Online First: 6-X-2015
}

D0l: http://dx.doi.org/10.15517/ijds.v0i0.21485

\section{RESUMEN}

El proceso de enseñanza-aprendizaje actual exige que las metodologías pedagógicas sean centradas en el estudiante y que se propicie el desarrollo de competencias como el trabajo colaborativo, análisis y resolución de problemas, entre otros aspectos. Por ello, el método del caso ofrece el análisis de una situación real, en un espacio académico que favorece la discusión entre pares y la aplicación de mayores exigencias cognitivas, lo que fortalece el aprendizaje significativo. El objetivo del presente trabajo es analizar la implementación del método de caso, mediante seminarios participativos, como un complemento académico al curso Clínica de Exodoncia y Cirugía de la Facultad de Odontología de la Universidad de Costa Rica.

\section{PALABRAS CLAVE}

Aprendizaje cirugía oral, Método del caso, Educación odontología. 


\section{ABSTRACT}

Modern teaching-learning processes demand student centered for educational methodologies, which develop skills such as collaborative work, analysis, problem solving, and others. The case method gives the opportunity to analyze a real situation in an academic environment, where students can discuss and apply higher cognitive requirements to relevant learning. The aim of this paper was to analyze the application of case method as participative seminars to supplement the Surgery Clinic course in the Faculty of Dentistry of University of Costa Rica.

\section{KEYWORDS}

Oral surgery learning, Method case, Dental Education.

\section{INTRODUCCIÓN}

Los estudiantes de Odontología de la Universidad de Costa Rica (UCR) reciben cursos teóricos y prácticos para desarrollar las competencias necesarias en el campo de la Cirugía Oral. La formación inicia en tercer año de carrera con los primeros cursos teóricos de Anestesia y Exodoncia; en cuarto reciben las primeras prácticas clínicas con un acompañamiento teórico en el curso de Cirugía Oral I. En quinto año, se complementa la enseñanza y práctica con los cursos de Clínica de Exodoncia y Cirugía I y II, y como teoría Cirugía Oral II (1).

La Clínica de Exodoncia y Cirugía es un curso práctico con refuerzo teórico, en el cual los estudiantes realizan exodoncias supervisadas por los docentes a cargo. Además, preparan dos presentaciones de caso para cirugía en las que deben analizar el compromiso sistémico del paciente, la metodología quirúrgica, farmacoterapia y las eventuales complicaciones que se podrían presentar; adicionalmente, los procedimientos quirúrgicos se realizan en forma demostrativa por el instructor. Debido a que los conocimientos teóricos son fundamentales para el desarrollo de la práctica clínica, estos se evaluan con una rúbrica durante las presentaciones de caso (2) y por medio, de tres pruebas escritas cortas sobre temas básicos desarrollados en cursos anteriores.
Sin embargo, la discusión y la ejecución de los casos quirúrgicos la realiza, individualmente, cada docente con un estudiante, por lo que el resto de los estudiantes pierde la oportunidad de aprendizaje, especialmente en casos quirúrgicos que, por su baja incidencia, es poco probable que puedan participar activamente del diagnóstico, tratamiento quirúrgico y manejo posoperatorio. Por lo tanto, es fundamental implementar estrategias didácticas con los casos quirúrgicos de interés docente, que involucren a toda la población estudiantil matriculada en la clínica. Por esta razón, el objetivo de este trabajo es analizar una implementación innovadora del método de caso mediante seminarios participativos, rescatando el trabajo de investigación individual que un estudiante realiza sobre un caso quirúrgico particular, que bajo la guía de su profesor se aproveche, teniendo como telón de fondo el problema patológico presentado, su solución y las posibles consecuencias, que eventualmente, podrían presentarse.

El método del caso es una estrategia pedagógica de amplia trayectoria; se utilizó por primera vez en 1914, en el programa de Derecho de la Universidad de Harvard, el objetivo fue recrear una situación real, con el fin de que los estudiantes aplicaran los conocimientos que poseían $n$ leyes para la solución del problema planteado. Este método ha evolucionado para 
desarrollar actividades pedagógicas en diversas ramas del conocimiento (3).

En el área de la salud es fundamental, porque existen cursos muy teóricos carentes del componente práctico que incentive el pensamiento crítico, por lo que esta estrategia brinda la oportunidad de resolver una situación real, sin la necesidad de estar frente al paciente, quien requiere una solución precisa y oportuna de sus padecimientos $(4,5,6)$; brindando al estudiantado la oportunidad de analizar detenidamente ese caso y aplicar sus conocimientos previos para enfrentar, resolver la patología y discutir posibles errores cometidos o diferentes abordajes, con el objetivo de que el estudiante enfrente adecuadamente situaciones similares que se le podrían presentar en su práctica profesional (7).

El método del caso también promueve las destrezas para evaluar integralmente al paciente y estimular la toma de decisiones clínicas siguiendo el método científico $(6,8)$. De manera que la «queja principal» por la cual el paciente se presentó, o sea, su patología, se convierta en el tema de estudio, que se aborda como una investigación clínica para completar la anamnesis, examen clínico y la revisión de literatura científica, a fin de brindar un diagnóstico presuntivo 0 hipótesis que deberá comprobarse 0 rechazarse, de acuerdo con exámenes diagnósticos como pruebas de laboratorio, imagenología y estudios histopatológicos, entre otros; y, finalmente, para establecer el diagnóstico definitivo y el tratamiento correspondiente $(6,8)$.

Esta estrategia requiere que el docente busque un «caso» que, desde la percepción académica, deberá definirse como la narración de una situación real que genera una oportunidad de aprendizaje, pues se requiere resolver una problemática (9). El grado de complejidad depende de los objetivos planteados para el curso en cuestión, ya que el docente deberá analizar los conceptos y técnicas, incluyendo los conocimientos previos requeridos para la resolución del caso (6, 9).La narración o introducción al caso debe estimular positivamente al estudiante, por lo tanto, la presentación debe ser creativa, para incentivar y motivar su participación $(3,9,10)$.

El docente tendrá una función secundaria, pues actuará como facilitador, moderador y orientador; en contraparte, el estudiantado tendrá mayor acción, ya que deberá empoderarse de su proceso de aprendizaje, por lo que se favorece el autoaprendizaje y el aprendizaje colaborativo $(3,9,10)$. Esta estrategia se desarrolla en varias etapas, en la primera, cada estudiante analiza los datos presentados y propone una hipótesis; posteriormente, se promueve la discusión grupal, la cual es fundamental, pues permite el aprendizaje entre pares, ya que cada uno explica su propuesta, expresa sus experiencias y discute conceptos, y finalmente del conjunto surge la resolución del caso (9).

Esta estrategia didáctica obliga al estudiante a sintetizar, analizar y evaluar; lo cual promueve un aprendizaje más significativo, porque utiliza un proceso cognitivo de mayor complejidad, que estimula realizar conexiones con las diversas ramas del conocimiento adquirido, para resolver el caso planteado, que deberá enfocarse como una situación real, tal como enfrentará en su vida profesional $(6,11,12,13,14)$. Asimismo, el método del caso favorece el desarrollo de habilidades, como la capacidad de síntesis, pensamiento crítico, análisis, trabajo colaborativo para la toma de decisiones y resolución de problemas, las cuales son competencias indispensables que deberían impulsarse en el estudiantado pues, actualmente, se requiere de profesionales con integridad, capaces de satisfacer las exigencias de una sociedad globalizada, basada en el conocimiento $(2,7,9,11,13,14,15)$.

Es fundamental que los docentes, con actividades académicas como la propuesta en 
este artículo, puedan inculcar al estudiantado las destrezas necesarias para desarrollar un proceso investigativo, orientado a la resolución de problemas. Por esta razón, el objetivo de la presente investigación es analizar la aplicación de un seminario participativo, basado en el método del caso.

\section{MATERIALES Y MÉTODOS}

La presente investigación es un estudio descriptivo bajo el paradigma cualitativo, pues permite recolectar información sobre un fenómeno social, desde el propio contexto donde se desarrolla (16), como es el análisis de la percepción de los estudiantes respecto a la aplicación del método del caso. Este enfoque tiene la ventaja que los datos recabados, al ser interpretaciones sociales, se recolectan mediante entrevistas 0 cuestionarios abiertos, que le permiten al grupo estudiado plasmar sus experiencias, percepciones y sugerencias, mientras está inmerso en la situación estudiada (16). Para tal efecto, se realizó un formulario con preguntas abiertas, donde el estudiante participante en el proceso del caso clínico describe sus percepciones sobre la actividad y su opinión respecto de si es adecuada o no esta metodología y su importancia para la futura implementación de un seminario como parte del curso.

La aplicación del método del caso en cuestión se realizó durante el II semestre del año 2013. Se inició con el análisis y desarrollo del caso quirúrgico, de un paciente que presentaba un odontoma compuesto, el cual es un tumor odontogénico benigno (Ver Figura 1). Una estudiante se encargó del proceso clínico y realizó la investigación sobre el diagnóstico, plan de tratamiento y manejo quirúrgico, según el protocolo establecido para los casos quirúrgicos.
Seguidamente, se programó la ectomía quirúrgica del tumor, la cual realizó el profesor en forma demostrativa con la estudiante en cuestión y se definió el seguimiento posoperatorio. Bajo condiciones normales, el proceso terminaría aquí; sin embargo, esta experiencia se compartió con el resto de estudiantes, invitados a participar en el seminario, cuya asistencia era voluntaria, y para motivarlos se les reconoció 15 puntos en el rubro de teoría, equivalente, según el programa actual del curso, a un trabajo escrito extra. Además, se les solicitó la firma de un consentimiento informado, en el cual se les explicó el objetivo de la actividad y la importancia de sus percepciones tanto positivas como negativas, pues dependiendo de estas, se implementaría una actividad similar para enriquecer el aprovechamiento académico del curso Clínica de Exodoncia y Cirugía. El seminario lo coordinó el docente que desarrolló el caso quirúrgico quien fungió como moderador y organizador, en contraparte, el estudiante que presentó el caso, actuó activamente brindando la información requerida, según la etapa de la actividad, pues el seminario participativo se subdividió en siete etapas para facilitar su desarrollo:

- Presentación del caso clínico: El docente introduce al estudiante expositor, quien brinda los datos generales del paciente, historia clínica, examen clínico y examen radiográfico.

- Diagnóstico presuntivo: El docente facilita una guía, en la cual cada estudiante participante, en forma individual y analizando la información suministrada, indica el diagnóstico presuntivo y menciona si se requieren exámenes complementarios para proseguir con el desarrollo del caso.

- Presentación del proceso diagnóstico: El estudiante expositor continúa explicando cómo se realizó el proceso diagnóstico, analiza los datos del paciente y las imágenes diagnósticas. 
- Plan de tratamiento inicial: El docente solicita que individualmente definan el plan de tratamiento, que incluye el abordaje quirúrgico y las posibles complicaciones que se pueden presentar (10 a 15 minutos).

- Plan de tratamiento discutido: El docente solicita que se organicen en grupos de 4 a 5 estudiantes, para que discutan el plan de tratamiento propuesto por cada uno. (10 a 15 minutos).

- Presentación final del caso: El estudiante presenta el tratamiento realizado.

- Discusión: Los grupos discuten las similitudes y diferencias con la resolución del caso propuesto por los grupos y el docente realiza un comentario para concluir la actividad.

El análisis de la información recabada, acorde con la metodología de los estudios cualitativos, se agrupó por semejanzas para interpretar la información según el contexto donde se desarrolló la actividad analizada. Las preguntas sobre la metodología actual y una futura implementación del método del caso se analizaron por medio de estadística descriptiva.

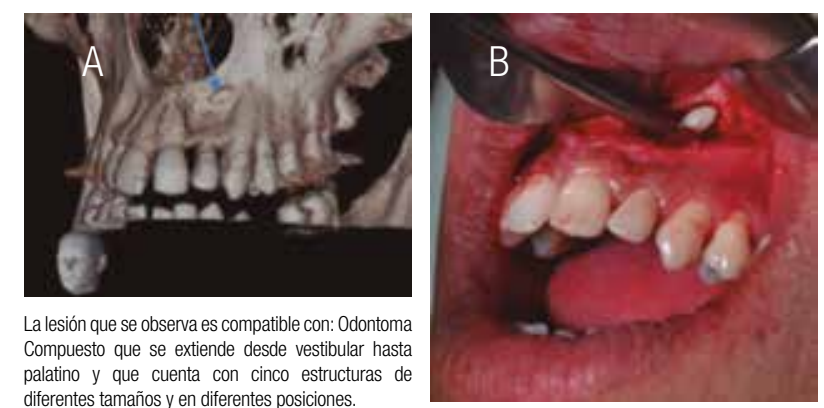

Figura 1. Imágenes de caso clínico empleado durante el seminario. A. Reconstrucción tridimensional de la tomografía axial computarizada donde se señala la ubicación del odontoma compuesto. B. Fotografía clínica durante la remoción de la patología.

\section{RESULTADOS}

Al seminario participativo, donde se aplicó el método del caso, asistieron 36 (72\%) estudiantes de los 50 matriculados, en el curso de Clínica de Exodoncia y Cirugía. La totalidad del estudiantado contestó positivamente acerca del desarrollo del seminario y, de igual forma, cuando se les consultó si esta actividad académica debería incorporarse como parte de la Clínica de Exodoncia y Cirugía. Los estudiantes describieron la actividad como provechosa, pues tenían que aplicar conocimientos anteriores a una situación real, también les gustó la interacción con los compañeros y el poder participar de un caso clínico que durante el desarrollo del curso no pudieron observar.

En cuanto al abordaje actual de la teoría en la Clínica de Exodoncia y Cirugía, 10 estudiantes (28\%) contestaron que era adecuado y 26 (72\%) lo contrario; cabe recalcar que el $100 \%$ del alumnado expresó que el seminario en el que participaron debería ser parte de la clínica.

\section{DISCUSIÓN}

Este es un estudio pionero, enfocado al empleo del método del caso en la Sección de Cirugía Oral y Maxilofacial de la Facultad de Odontología, aunque presenta un sesgo, debido a que por participar, los estudiantes recibieron 15 puntos extra en su calificación; no obstante, se debe resaltar el efecto positivo, pues voluntariamente asistió el $72 \%$ de los estudiantes que se encontraban matriculados en el curso y se recopiló su percepción sobre la actividad realizada; posiblemente aquellos que no participaron presentan características distintas, incluyendo un menor interés por actividades extracurriculares.

Debido a la respuesta positiva obtenida en el seminario, en el que se desarrolló el método del caso, se deberán realizar las gestiones para incorporar un seminario a la Clínica de Exodoncia y Cirugía, para amalgamar los conocimientos teóricos desarrollados en cursos anteriores con la práctica quirúrgica, de manera que sirva como un espacio interactivo para analizar casos clínicos de patologías orales, técnicas quirúrgicas y tratar temas básicos que frecuentemente se olvidan, pero que podrían fortalecerse por medio del 
desarrollo de un caso clínico real, el cual exige un planteamiento del problema, síntesis de datos importantes, el análisis y la solución del problema; actividades que engloban una realidad del ejercicio profesional $(7,9,10,11,15)$.

Es importante implementar la metodología propuesta en un futuro curso, pues investigaciones realizadas revelaron que el estudiantado desarrolla tanto habilidades analíticas como competencias comunicativas, lo que fomenta el trabajo colaborativo y la motivación entre compañeros, y refuerza el proceso de aprendizaje y la educación continua (4, $5,14)$ que, a su vez, mejora la confianza en los conocimientos aprendidos.

Asimismo, la aplicación de esta metodología didáctica ha demostrado mejores resultados que la discusión de libros de texto, también incrementa las habilidades en la comunicación verbal y escrita; además, motiva el aprendizaje en los estudiantes, quienes refieren, a su vez, sentir mayor confianza en los conocimientos aprendidos $(5,13,14,17)$.

Por otro lado, es importante que el profesorado participante se comprometa en este cambio de paradigma educativo, centrado en el estudiante, porque el método exige que el docente se transforme en orientador, motivador y guía, para lo cual debe desarrollar competencias pedagógicas apropiadas $(5,9,13,18)$.

En futuras investigaciones es necesario analizar, tanto la percepción docente como evaluar su desempeño, pues es una metodología centrada en el estudiante que requiere profesores con cualidades diferentes a la pedagogía tradicional. Además, es fundamental el desarrollo de estudios que permitan crear metodologías de evaluación acordes con la estrategia didáctica expuesta e inclusive adaptarlas al medio electrónico.

\section{CONCLUSIONES}

La utilización del método del caso como estrategia pedagógica es fundamental en el proceso de enseñanza-aprendizaje de los estudiantes de Odontología y de otras ramas del área de la salud, pues brinda una oportunidad de acercamiento a situaciones reales que no se presentan frecuentemente durante los cursos prácticos. Asimismo, la incorporación de un seminario en la Clínica de Exodoncia y Cirugía es un espacio que le permite al estudiantado el desarrollo de competencias necesarias para solucionar los casos clínicos de su futura práctica profesional, también fortalece el trabajo colaborativo con sus compañeros. Por lo tanto, es imprescindible incorporar esta estrategia pedagógica en el curso de Clínica de Exodoncia y Cirugía y valorar su utilización en otros cursos.

\section{CONFLICTOS DE INTERÉS}

No existen conflictos de interés.

\section{REFERENCIAS}

1. Facultad de Odontología. Plan de estudios. San José, Costa Rica: Universidad de Costa Rica; 2014. 10p. Disponible en: http://www. fodo.ucr.ac.cr/node/29.

2. Hernández-Rivera P. ¿Por qué es importante establecer una rúbrica de evaluación? El caso del curso Clínica de Exodoncia y Cirugía. Revista Educación. 2012; 36(1): 63-72.

3. Dirección de Investigación y Desarrollo Educativo. Las estrategias y técnicas didácticas en el rediseño. Monterrey, México: Instituto de Estudios Superiores Monterrey; [s.f]. 37 p.

4. Laver S. Croxon L. Narrative pedagogy with evolving case study - A transformative 
approach to gerontic nursing practice for undergraduate nursing students. Nurse Educ Pract. 2015; 15(3): 1-4.

5. Rezaee R. Mosalanejad L. The Effects of Case-Based Team Learning on Students' Learning, Self Regulation and Self Direction. Glob J of Health Science. 2015; 7(4): 295-306.

6. Camacho Carr K. Using the unfolding case study in midwifery education. J Midwifery Womens Health. 2015. doi:10.1111/ jmwh.12293.

7. Amaya Afanador A. Simulación clínica: aproximación pedagógica de la simulación clínica. Universitas Méd. 2010; 51(2): 204-211.

8. Ilizástigui-Dupuy F. Rodríguez-Rivera L. El método clínico. MediSur. 2010; 8(5): 2-11.

9. Rosker EJ. El método de casos como herramienta transformadora de la sociedad. Universidad \& Empresa. 2006; 5 (11): 109-122.

10. Chaparro Gómez LE. Uso de los casos clínicos como estrategia didáctica en la enseñanza de anestesia. Revista Colombiana de Anestesiología. 2004; 32(4): 281-284.

11. Dutra D. Implementation of case studies in undergraduate didactic nursing courses: a qualitative study. BMC Nurs. 2013; 12: 15-24.

12. Nentl N. Zietlow R. Using Bloom's Taxonomy to Teach Critical Thinking Skills to Business Students. Coll Undergrad Lib. 2008; 15 (1-2): 159-172.

13. Bonney KM. Case Study Teaching Method Improves Student Performance and Perceptions of Learning Gains. J Microbiol Biol Educ. 2015; 16(1): 21-28.

14. Kantar LD. Massouh A. Case-based learning: What traditional curricula fail to teach. Nurse Educ Today. 2015; 35(7): e8-e14.

15. Camacho Gómez M. Historias empresariales en los estudios de casos. Pensam Gest. 2011; 31: 196-210.

16. Hernández R. Fernández C. Baptista P. Metodología de la Investigación. México: Mc Graw Hill Interamericana; 2003. 705p.

17. Tolchin B. Willey JZ. Prager K. Education research: a case-based bioethics curriculum for neurology residents. Neurology. 2015 Mar; 84(13): e91-e93.

18. Salazar Botello C.M., Chiang Vega M. Competencias y educación superior. Un estudio empírico. Horizontes Educacionales. 2007; 12(2): 23-35. 